\title{
EpidemicSim: Epidemic Simulation System with Realistic Mobility
}

\author{
Steven Kopman, Mustafa Ilhan Akbaş and Damla Turgut \\ Department of Electrical and Computer Engineering \\ University of Central Florida \\ Orlando, FL 32816 \\ Email: \{skopman,miakbas,turgut $\} @$ eecs.ucf.edu
}

\begin{abstract}
Much attention has been paid to modeling human behavior and social interactions for epidemic and pandemic predictions. Nearly all of these models and predictive simulations rely on synthetic individuals to simulate social patterns using data gathered from databases such as census and transportation information. At the same time the ubiquity of mobile devices and online social networks have created an opportunity for real-life simulations of disease transmissions.

In this paper, we lay the groundwork for a mobile epidemic simulation framework by creating a simulation model of individuals walking in a defined space with varying durations. The population size and walking area are varied to determine the best parameters for simulating disease spread. The effects of different infection probabilities are also investigated for a more realistic simulation framework. The resulting information is employed to create disease social networking maps used to determine the importance of each individual in the network and the connections between the ground zero source of the disease and the total infected population at the end of the simulation.
\end{abstract}

\section{INTRODUCTION}

Accurate prediction of epidemic and pandemic diseases is essential to preventing both infections and deaths related to each modeled disease. The Severe Acute Respiratory Syndrome (SARS) outbreak in 2003 affected over two dozen countries in four continents. The SARS virus only infected 8,098 people worldwide, with 774 dying [1], but the threat of its spread caused great panic around the world. The 2009 H1N1 pandemic, also known as Swine Flu, infected an estimated 61 million people in the United States with 12,470 deaths [2]. This disease led to a widespread push for vaccinations of children and the elderly. However, the prediction of the spreading characteristics of H1N1 was difficult.

Besides these highlighted pandemics, the spreading of common cold and flu viruses are also critical to analyze. There are over one billion colds in the United States each year with children averaging three to eight colds per year [3]. All of these examples emphasis the need to model and predict the spread of similar diseases.

Each of the discussed viruses and bacterial infections has different requirements for transmission, ranging from physical contact to being in the same room as an infected person. Additionally, each one has a probability of being transmitted when a particular person-to-person contact distance has been met. Hence these parameters mean that a single type of sensor can not be used to estimate the transmission of the disease.
Specific properties of individuals such as the age, sex and the health status also must be used to evaluate their probabilities to get infected. Moreover, the characteristics of particular social group, in which the disease spreads, and its environment are also critical when determining the infection probabilities.

In this paper, we utilize a disease model to define the necessary parameters, such as population size and area, required for the implementation of the mobile version of our modeling framework for epidemic diseases, the EpidemicSim system. This information lays the groundwork for utilizing the ubiquity of mobile devices as the modeling platform. Simulating these requirements will prove the feasibility of using real-life human interactions as a unique disease modeling platform.

The number of social connections people have in the network is also employed to evaluate each user's role in the spread of disease. Metrics such as Betweenness, Degree, Centrality and Bridges [4] are critical to understand the importance of each individual on the transmission of the disease. The combination of these values defines various characteristics of individuals such as the probability of being responsible for the transmission of the disease to a specific community or the popularity in terms of getting infected. Therefore, the number of connections, the number of hubs and bridges in the network are also analyzed to have a better model for the framework. The goal is to utilize this system in an open, yet well defined environment such as a university campus where a maximum number of interactions can occur and an accurate social network map can be produced.

The combined information of life-like human interaction with disease spread simulation can provide a unique platform for the modeling of human behavior and interaction. Therefore mobility model used to simulate the movement of people is critical in simulations. Our system uses a realistic mobility model, based on Lévy walk, to simulate the movement of people in the simulation area. The proximity data and the interaction information used in the system depend on this mobility model.

The rest of the paper is organized as follows. Section II summarizes the related work. We provide a detailed description for our approach in Section III. We show the simulation results in Section IV and finally conclude in Section V. 


\section{RELATED WORK}

Disease simulations employ mathematical models to estimate the probability of infection and spread. These models are generally applied to synthetic individuals on powerful computers to emulate real-life social interactions and movements. The generation of accurate synthetic individuals can be accomplished by using databases of information such as census data or transportation and models from other domains such as social and economic domains. The models must be scalable and configurable to allow for different diseases to be introduced to multiple different environments.

The Synthetic Environments for Analysis and Simulation (SEAS) [5] technology developed by Purdue University and licensed to Simulex uses agent based modeling to simulate a virtual world. The artificial agents represent fine detailed decision making while real human players represent the countries, firms, and so on in the rest of the virtual world. These models have been used to simulate biological attacks in the BioReady case study.

The Los Alamos National Laboratory has also developed a stochastic, agent-simulation engine called EpiSimS [6]. The EpiSimS model uses 20 million synthetic individuals per region, with the United States divided into 15 regions. Each individual's activity model is derived from the TRANSIMS [7] transportation simulation code. TRANSIMS is used to analyze traffic movement by the U.S. Department of Transportation but its mixing patterns are consistent with person-to-person social networks. EpiSimdemics [8] by Barrett et al. is a parallel algorithm, which simulates the spread of a disease in large social networks. EpiSimdemics uses individual-based models and scales up to social networks with 100 million individuals by exploiting the semantics of disease evolution and propagation.

Both of these systems rely on modeled behavior from databases and other model inputs to generate a virtual world of individuals. All of the movements and interactions are simulated as near as possible to real life. EpiSimS also provides limited social networking results in a cone shape from the red $X$ in the tip to the base of the cone.

Real life disease spread simulations have been proposed and implemented by a team at the University of Cambridge. The FluPhone [9] application uses a smartphone's Bluetooth connection to detect other Bluetooth devices nearby and send back the information to the server. It also asks participants to record their flu symptoms, if any. The application also can be extended to simulate virtual disease outbreaks. The use of Bluetooth for ranging information is the main limitation of the system as it can extend tens of meters from the transmitting source. Yoneki et al. [10] presented a study of epidemic spread in dynamic human networks from real connectivity traces. The data used in this study include the traces taken from the Cityware project, in which there are nine Bluetooth scanners deployed across the city of Bath, England. These devices were deployed to monitor the presence of mobile devices within a ten metre radius [11]. The proximity of the devices are determined by using the log data and some of the devices used Bluetooth scanning programs [12] to collect the detected device information via GPRS. The authors show the effects of periodic human activity, lifetime of the virus and different types of social hub nodes on the epidemic spread.

The underlying network and the movement of the nodes are critical to model the disease spread. The spread of viruses in plant populations [13], in animal societies [14] and even in digital networks share important characteristics and modeled similarly. The travel models in a city [15] or within the worldwide airline network [16] are studied for this purpose. We consider people moving in an environment such as a university campus in our simulations. There are various human mobility models presented in the literature. The random walk model group is one of these, in which the next destination of a node and the velocity are chosen randomly based on the probability distribution. Random Waypoint (RWP) [17] serves as the base of this group and has been extended for many other models [18]. However, random walk models are not suitable for human mobility in realistic scenarios. Human mobility has several characteristic features, which have been observed by different measurement methods. Examples of these features are truncated power-law distributions of pause times, intercontact times, fractal waypoints and heterogeneously defined areas of individual mobility. Rhee et al. [19] shows that these properties are similar to the features of Lèvy walks and used these properties to design Truncated Lévy Walk (TLW) model, which is a random equivalent mobility model for human walks. TLW is used in our simulations to create a realistic human mobility.

\section{EPIDEMICSIM SYSTEM}

The EpidemicSim system is modeled to validate the concepts presented. The three main components used in MATLAB [20] includes the mobility model, interaction computation, and the network map.

The first component is the mobility model for human mobility. This is needed to simulate the walking paths of the individuals across a defined area. The walking path model is planned to be replaced by the user carrying a smartphone in future implementations.

The second component is the distance measuring and interaction computation. For each time period defined in the walking model, the pairwise distance between each individual must be calculated. If two individuals are within the defined infection distance, their susceptibility and infection values are checked. An infected individual will only infect a susceptible individual. This basic concept will be carried forward to the mobile version of the system. However, each disease will define its infection probability and infection range requirements. The smartphone's sensors will be used to apply these requirements.

The third component of the simulation model, the network map, is used to aid in infection analysis using social networking metrics. This component will run on a backend server 
in the future system and will provide further analysis of the infection network.

\section{A. Mobility model}

Lévy walk is an optimal way to find randomly dispersed objects [21]. Lévy walk is observed as the mobility model in most of the animal foraging patterns. It is shown that human mobility also can be modeled by Lévy walk model [19]. Lévy walk is a random walk with step-lengths distributed according to a heavy-tailed probability distribution. Lévy walks are Markov processes and after a large number of steps, the distance from the origin of the random walk tends to be reach stable distribution. Lévy distribution is the Fourier transformation of the moving distance of a single random walk and Rhee et al. [19] gives its PDF as follows:

$$
f_{z, \alpha}(x)=\frac{1}{2 \pi} \int_{-\infty}^{\infty} e^{-i z t} \phi(t) d t
$$

where $\phi(t)=e^{-|C t|^{\alpha}}$ and $C$ is a constant.

The distribution can be approximated by a power law of the form $y=x^{-\alpha}$ where $0<\alpha<2$. Each step in Lévy walk can be expressed by a tuple $L=\left(1, \theta, \Delta t_{f}, \Delta t_{p}\right)$. $\Delta t_{f}$ indicates the duration and it is chosen for each walk from a probability distribution $P(l) . \Delta t_{p}$ specifies pause time at the end of a walk and $\theta$ is the random direction taken by a node. A Lévy walk contains many short walks and a small number of long walks. The resulting pattern depends highly on the value of $\alpha$ used in the system. As $\alpha$ becomes greater, the number of short walks increases.

The TLW mobility model for our simulation uses the alpha and beta values of 0.88 and 1 . The square areas of $1000 \times 1000$ and $2500 \times 2500$ are also used and together with the alpha and beta values, these parameters correspond to a college campus. The distances are unitless for the TLW model. However, we will refer to them in feet for an easy reference.

An example of a social network map for $1000 \times 1000$ square distance is given in Fig. 3. Each individual moves at 30 second intervals and is allowed to pause for a minimum of 30 seconds and a maximum of 600 seconds. The number of individual paths and the duration of each run is varied to allow the evaluation of multiple scenarios within one test environment.

\section{B. Distance Measurement and Interaction Computations}

The TLW model produces one path for each individual over a fixed duration. A path consists of $x$ and $y$ coordinates for each 30 second interval. The $x y$ coordinates are used to generate the distance between each individual, pairwise, at each time interval. The used distance formula is as follows:

$$
\operatorname{distance}\left(I_{1}, I_{2}, t\right)=\sqrt{\left(x_{1}(t)-x_{2}(t)\right)^{2}+\left(y_{1}(t)-y_{2}(t)\right)^{2}}
$$

where $I_{1}$ and $I_{2}$ are the locations of two individuals at time $t$.

The individual positions at each interval are stored in a location matrix is also an input to the interaction component. The interaction component determines whether two individuals met during a time interval. For the purpose of this simulation, two nodes are interacting if they are within ten feet of each other during the interval. This value was chosen as part of the disease model and is an input to the system, allowing for the future application of a more complex disease model.

\section{Disease Model and Mapping}

The pairwise analysis of the distances between each individual at all time intervals provides the basis for the disease model. For the simulation of a disease spread in the population, a random individual is chosen to be the initial carrier for the disease.

The status of each individual is tracked using two attributes. The first attribute is whether a node is susceptible to being infected by the disease. All individuals, except the initial infected person, begin in this state. The second attribute is whether a node is infected. If a node comes into contact with an infected node and it is susceptible (not infected), it will be infected. This approach is similar to the standard Susceptible, Infectious, Recovered (SIR) epidemic model [22]. However for our system, individuals in the simulation do not enter the recovered state.

In addition to the infected state, each individual determined to be infected is also tagged with the id of the individual that infected the person. This method provides information on the social interactions among all of the individuals in the network. The interaction matrix that is a result of the application of the disease model to the TLW paths is used to generate a network map as well as to provide the basis for social networking analysis. These results are presented in Section IV.

\section{Simulation Study}

The simulations are conducted by using the model described in Section III and they are divided in two main groups. In the first group, the probability of infection is taken as $100 \%$ when two people contact. In other words, when a susceptiple (not infected) person comes into contact with an infected person, the disease is transmitted. On the other hand, the second simulation set includes infection probability of the disease such that a susceptiple person is infected with a probability defined according to the disease when the person comes into contact with an infected person.

Table I summarizes the main simulation parameters used in our simulation study. The settings are chosen to match the requirements of the scenario. In each simulation, the number of individuals, the square range or the duration is varied. Two population size of 250 and 500 were considered in the simulations. Durations were 240 minutes (four hours) and 480 minutes (eight hours). The square ranges of $1000 \times 1000$ and $2500 \times 2500$ were used. Each run was evaluated for the number of infected people, the number of people infected by the initial infected person and the maximum number of people infected by a single individual. Additionally, the number of hubs, defined as a node with more than two connections, and bridge nodes, which are linking hubs, are also employed. 
TABLE I

SIMULATION PARAMETERS

\begin{tabular}{|l|l|}
\hline \multicolumn{1}{|c|}{ Parameter } & \multicolumn{1}{c|}{ Value } \\
\hline Small population size & 250 \\
\hline Large population size & 500 \\
\hline Small area size & $1000 \times 1000$ \\
\hline Large area size & $2500 \times 2500$ \\
\hline TLW $\alpha$ & 0.88 \\
\hline TLW $\beta$ & 1 \\
\hline Simulation time & $240-480$ mins \\
\hline Number of runs & 30 \\
\hline
\end{tabular}

\section{A. Simulation Results}

1) Simulation group 1: There are six simulation sets in the first simulation group and each of them had five runs with the parameters defined in Table I. Table II shows the results for all the simulation runs.

The initial simulations were run with a range size of $1000 \times 1000$. The population sizes used were 250 and 500 people for four hour duration. For both populations, almost the entire population was infected in most of the simulations. In only one case, $28 \%$ of the population remained uninfected.

The next two sets utilized the same population sizes and duration with a larger walking area. The square range of $2500 \times 2500$ was considered. The number of infected individuals during these runs decreased significantly compared to the sets with smaller walking areas. The runs of a population size of 250 individuals had 1 to 22 infected people. The infected people for the population size of 500 individuals ranged from 7 to 90 .

The combined results of the simulations are significant in showing that increasing the walking area reduced infections by nearly $95 \%$ in the small population and $88 \%$ in the larger population. This decrease is also significant because the disease model is simplistic and only a single time interval of contact within 10 feet causes an infection. In a real world epidemic model, diseases may not transfer as easily, thus reducing the infection rate even further.

In the final two simulations, the duration is increased to eight hours while keeping the population size constant at 500 individuals. One set utilized the square range of $1000 \times 1000$ and the other used the square range of $2500 \times 2500$. As in the runs with shorter durations, the smaller range yielded an infection rate of $100 \%$. When the area size was increased, the number of infected individuals dropped slightly to an average of 441 compared to 500 in the smaller range. In the previous set with the same population size and square range, the infection rate for a four hour duration averaged $12 \%$. When the duration was doubled, this value increased to $88.16 \%$.

2) Simulation group 2: The simulation groups in the second simulation set included an additional parameter, which is the probability of infection. The three different values used for the probability of infection are $20 \%, 50 \%$ and $70 \%$. Each simulation set has five runs with the probability of infection and the parameters defined in Table I. Table III shows the results of the simulation runs.
The simulations with population size of 250 in an area of $2500 \times 2500$ were run for four hours. Three different sets of simulations were run for all three different values of the infection probabilities to observe the effect of the infection probability on the results. Similar to the first set of simulations, the number of infected people, hubs and bridges are observed in these conditions.

The simulation runs for a population of 250 people with the varied infection probabilities are compared to the results of the simulation group 1 in Fig. 1. Simulation group 1 utilizes an effective infection probability of $100 \%$ as any contact automatically results in an infection. In all cases with a duration of four hours and a range of $2500 \times 2500$, the infection rate was low. Infection probabilities of $20 \%, 50 \%$ and $70 \%$ resulted in averages of less than three individuals. This compares with an infection average of 11.2 for the runs from group 1. Statisically this shows that with a large area and low population infection probability has little effect on the results. Fig. 2 shows that by doubling the population size, the infection rate increases approximately 10 fold for an infection probability of $20 \%$ and more than 20 fold for an infection probability of $70 \%$.

The effect of the infection probability is observed better when the population size is doubled and the area size is decreased to $1000 \times 1000$. In the first set of simulations with the same conditions, shown by the dark grey bar in Fig. 2 for the $100 \%$ probability, the whole population becomes infected at the end of the simulation period. When the infection probability of $20 \%$ is used, the average number of infected people decreases by $54.3 \%$. In particular simulation runs, the number of infected people is as low as 56 or 67 . However, on average, approximately half of the population becomes infected. When the infection probability is increased to $70 \%$, the results are equal to the results of the $100 \%$ probability group.

When the numbers of connections are compared, the difference is again significant. The numbers of hubs and bridges are greater than 115 and 106 respectively in all simulations for the first simulation group. When the infection probability of $20 \%$ is used, the numbers of hubs and bridges drop to minimum of 14 and 13 with the averages of 59.8 and 54.2. The average number of maximum connections also drops from 10.6 to 8.6. For the $70 \%$ sets, the maximum number of connections and the number of hubs and bridges are also very similar to the first simulation group. Therefore, we can conclude that in these conditions, the infection probability is effective only if it is a low value such as $20 \%$ mirroring the effect on the number of infections.

Simulating a simple disease model while applying modeled human mobility yielded important results that can be used in the future work to implement a real world epidemic simulation model. First, increasing the population size in a small walking area with a short duration has little effect as nearly all individuals became infected even with a small population. Second, when the walk space was increased by nearly six times with the same population density and duration, the infection 
TABLE II

EPIDEMICSIM SIMULATION RUNS

\begin{tabular}{|c|c|c|c|c|c|c|c|c|}
\hline${ }_{\#}^{\text {Run }}$ & $\begin{array}{l}\text { Population } \\
\text { Size }\end{array}$ & $\begin{array}{l}\text { Duration } \\
\text { (min) }\end{array}$ & Range & $\begin{array}{l}\text { Total } \\
\text { Infected }\end{array}$ & $\begin{array}{l}\text { Initial } \\
\text { Infected } \\
\text { Person } \\
\text { Connections } \\
\end{array}$ & $\begin{array}{l}\text { Max } \\
\text { Connections }\end{array}$ & $\begin{array}{l}\text { Hubs } \quad(>2 \\
\text { Connections) }\end{array}$ & $\begin{array}{l}\text { Bridges } \\
\text { (Link Two } \\
\text { or More } \\
\text { Hubs) } \\
\end{array}$ \\
\hline 1 & 250 & 240 & 1000 & 250 & 7 & 9 & 61 & 60 \\
\hline 2 & 250 & 240 & 1000 & 250 & 5 & 7 & 66 & 60 \\
\hline 3 & 250 & 240 & 1000 & 250 & 4 & 10 & 62 & 59 \\
\hline 4 & 250 & 240 & 1000 & 249 & 8 & 8 & 63 & 60 \\
\hline 5 & 250 & 240 & 1000 & 180 & 5 & 7 & 45 & 41 \\
\hline 1 & 250 & 240 & 2500 & 4 & 2 & 2 & 0 & 0 \\
\hline 2 & 250 & 240 & 2500 & 17 & 2 & 4 & 3 & 2 \\
\hline 3 & 250 & 240 & 2500 & 1 & 0 & 0 & 0 & 0 \\
\hline 4 & 250 & 240 & 2500 & 12 & 4 & 4 & 3 & 3 \\
\hline 5 & 250 & 240 & 2500 & 22 & 4 & 4 & 4 & 2 \\
\hline 1 & 500 & 240 & 1000 & 500 & 9 & 10 & 122 & 113 \\
\hline 2 & 500 & 240 & 1000 & 500 & 8 & 11 & 115 & 109 \\
\hline 3 & 500 & 240 & 1000 & 500 & 12 & 14 & 118 & 106 \\
\hline 4 & 500 & 240 & 1000 & 500 & 5 & 8 & 124 & 114 \\
\hline 5 & 500 & 240 & 1000 & 500 & 3 & 10 & 115 & 108 \\
\hline 1 & 500 & 240 & 2500 & 80 & 6 & 8 & 13 & 11 \\
\hline 2 & 500 & 240 & 2500 & 32 & 4 & 5 & 7 & 6 \\
\hline 3 & 500 & 240 & 2500 & 78 & 4 & 9 & 18 & 15 \\
\hline 4 & 500 & 240 & 2500 & 7 & 4 & 4 & 1 & 0 \\
\hline 5 & 500 & 240 & 2500 & 99 & 3 & 5 & 25 & 23 \\
\hline 1 & 500 & 480 & 1000 & 500 & 4 & 9 & 123 & 113 \\
\hline 2 & 500 & 480 & 1000 & 500 & 7 & 10 & 133 & 124 \\
\hline 3 & 500 & 480 & 1000 & 500 & 7 & 8 & 126 & 116 \\
\hline 4 & 500 & 480 & 1000 & 500 & 9 & 9 & 132 & 125 \\
\hline 5 & 500 & 480 & 1000 & 500 & 8 & 10 & 129 & 120 \\
\hline 1 & 500 & 480 & 2500 & 483 & 9 & 9 & 118 & 111 \\
\hline 2 & 500 & 480 & 2500 & 485 & 9 & 10 & 123 & 116 \\
\hline 3 & 500 & 480 & 2500 & 452 & 4 & 9 & 118 & 112 \\
\hline 4 & 500 & 480 & 2500 & 289 & 6 & 7 & 77 & 73 \\
\hline 5 & 500 & 480 & 2500 & 495 & 6 & 8 & 119 & 110 \\
\hline
\end{tabular}

rate was significantly less, as expected. Finally, when the duration was doubled, the infection rate returned to nearly the same level as the small area tests with the same population size. Therefore, when the walk area is very large with the relatively small population, the duration must be increased significantly to allow for adequate coverage by all individuals. When the walk space is relatively small, the population size is not as significant and increasing duration may not be required. Finally, if the walk area is very large and a longer duration is not possible, similar results can be produced by capping the walk space individuals can use. Since humans may not walk exactly as they do in the model, this information may need to be adjusted when the system is ported to mobile devices.

\section{B. Network Maps and Social Analysis}

In each simulation, the social map of the network is drawn according to the proximity data gathered from the simulation. Fig. 3 shows an example social network map of infection spread in a network of 500 nodes in an area of $1000 \times 1000$. The uninfected nodes throughout the simulation time are not included in the figure.

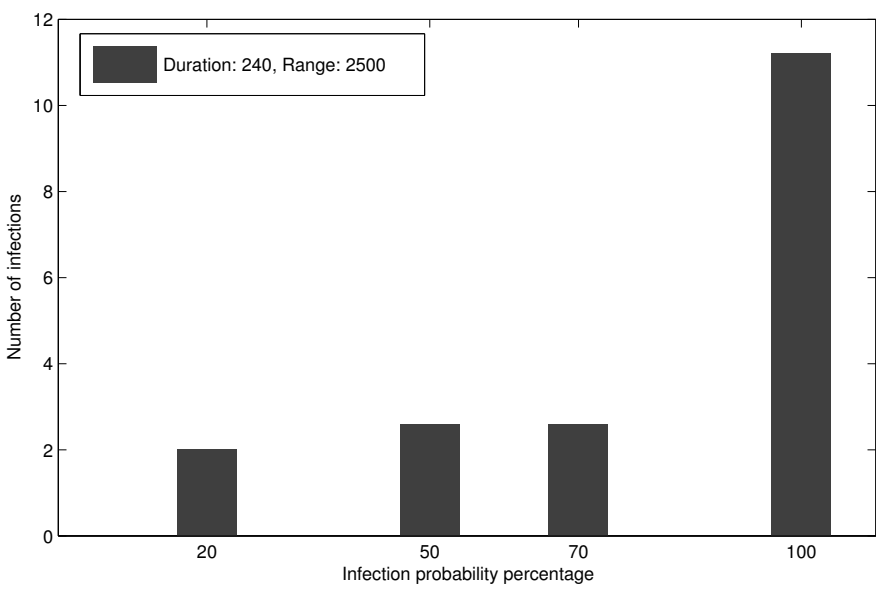

Fig. 1. Infections vs. Infection Probability - 250 People - Sets 1 and 2

The number of connections each node has over the life time of the simulation shows the importance and the popularity of the node. The nodes with high total connections in the network map are the high degree hub nodes. The critical effect of the 
TABLE III

EPIDEMICSIM SIMULATION RUNS WITH INFECTION PROBABILITIES

\begin{tabular}{|c|c|c|c|c|c|c|c|c|c|}
\hline $\begin{array}{l}\text { Run } \\
\#\end{array}$ & $\begin{array}{l}\text { Population } \\
\text { Size }\end{array}$ & $\begin{array}{l}\text { Duration } \\
\text { (min) }\end{array}$ & Range & $\begin{array}{l}\text { Total } \\
\text { Infected }\end{array}$ & $\begin{array}{l}\text { Initial } \\
\text { Infected } \\
\text { Person } \\
\text { Connections }\end{array}$ & $\begin{array}{l}\text { Max } \\
\text { Connections }\end{array}$ & $\begin{array}{l}\text { Hubs (>2 } \\
\text { Connections) }\end{array}$ & \begin{tabular}{l}
\multicolumn{2}{l}{ Bridges } \\
(Link Two \\
or More \\
Hubs)
\end{tabular} & $\begin{array}{l}\text { Infection } \\
\text { probability }\end{array}$ \\
\hline 1 & 250 & 240 & 2500 & 1 & 0 & 0 & 0 & 0 & $20 \%$ \\
\hline 2 & 250 & 240 & 2500 & 3 & 1 & 2 & 0 & 0 & $20 \%$ \\
\hline 3 & 250 & 240 & 2500 & 2 & 1 & 1 & 0 & 0 & $20 \%$ \\
\hline 4 & 250 & 240 & 2500 & 2 & 1 & 1 & 0 & 0 & $20 \%$ \\
\hline 5 & 250 & 240 & 2500 & 2 & 1 & 1 & 0 & 0 & $20 \%$ \\
\hline 1 & 250 & 240 & 2500 & 3 & 1 & 2 & 0 & 0 & $50 \%$ \\
\hline 2 & 250 & 240 & 2500 & 6 & 2 & 2 & 0 & 0 & $50 \%$ \\
\hline 3 & 250 & 240 & 2500 & 1 & 0 & 0 & 0 & 0 & $50 \%$ \\
\hline 4 & 250 & 240 & 2500 & 1 & 0 & 0 & 0 & 0 & $50 \%$ \\
\hline 5 & 250 & 240 & 2500 & 2 & 1 & 1 & 0 & 0 & $50 \%$ \\
\hline 1 & 250 & 240 & 2500 & 4 & 2 & 2 & 0 & 0 & $70 \%$ \\
\hline 2 & 250 & 240 & 2500 & 1 & 0 & 0 & 0 & 0 & $70 \%$ \\
\hline 3 & 250 & 240 & 2500 & 4 & 2 & 2 & 0 & 0 & $70 \%$ \\
\hline 4 & 250 & 240 & 2500 & 2 & 1 & 1 & 0 & 0 & $70 \%$ \\
\hline 5 & 250 & 240 & 2500 & 2 & 1 & 1 & 0 & 0 & $70 \%$ \\
\hline 1 & 500 & 240 & 1000 & 425 & 9 & 9 & 122 & 111 & $20 \%$ \\
\hline 2 & 500 & 240 & 1000 & 280 & 5 & 9 & 75 & 68 & $20 \%$ \\
\hline 3 & 500 & 240 & 1000 & 314 & 5 & 11 & 71 & 63 & $20 \%$ \\
\hline 4 & 500 & 240 & 1000 & 56 & 4 & 8 & 14 & 13 & $20 \%$ \\
\hline 5 & 500 & 240 & 1000 & 69 & 6 & 6 & 17 & 16 & $20 \%$ \\
\hline 1 & 500 & 240 & 1000 & 500 & 6 & 10 & 128 & 125 & $70 \%$ \\
\hline 2 & 500 & 240 & 1000 & 500 & 8 & 12 & 125 & 113 & $70 \%$ \\
\hline 3 & 500 & 240 & 1000 & 500 & 9 & 12 & 126 & 112 & $70 \%$ \\
\hline 4 & 500 & 240 & 1000 & 500 & 6 & 13 & 124 & 117 & $70 \%$ \\
\hline 5 & 500 & 240 & 1000 & 500 & 11 & 11 & 124 & 115 & $70 \%$ \\
\hline 1 & 500 & 240 & 2500 & 30 & 4 & 5 & 7 & 6 & $70 \%$ \\
\hline 2 & 500 & 240 & 2500 & 28 & 2 & 7 & 5 & 4 & $70 \%$ \\
\hline 3 & 500 & 240 & 2500 & 60 & 4 & 10 & 13 & 12 & $70 \%$ \\
\hline 4 & 500 & 240 & 2500 & 22 & 4 & 7 & 3 & 3 & $70 \%$ \\
\hline 5 & 500 & 240 & 2500 & 2 & 1 & 1 & 0 & 0 & $70 \%$ \\
\hline
\end{tabular}

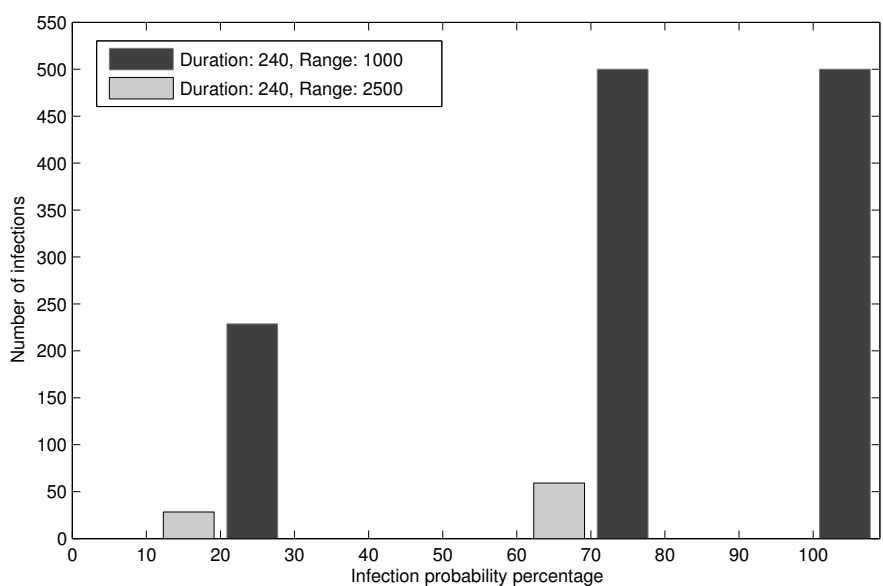

Fig. 2. Infections vs. Infection Probability - 500 People - Sets 1 and 2

hub nodes can be observed in Fig. 3, which gives the disease social network map from the simulation of 500 individuals in an area of $1000 \times 1000$. The nodes represent the infected individuals, the links represent the transmission of the disease.
Uninfected individuals are not represented in the figure.

The transmission of the disease is mainly through the hub nodes in our framework, which forms a realistic feature of our simulation model. One of the interesting results of the simulations shows that although the first person is the central source of the infection, that person is generally not the highest order hub in the simulation.

In all but one of the simulations in the area of $1000 \times 1000$, the initial person did not have the most connections. Additionally, an increase in the population size in this scenario does not have a major effect on the number of connections the initial and highest order hubs have. The connection number range for a population of 250 was four to eight for initial hubs and seven to ten for highest order hubs. When the population was increased to 500, the values changed to three to twelve and eight to fourteen. The major difference was the doubling of the number of hubs and bridges, from an average of $23.76 \%$ and $22.40 \%$ to $47.52 \%$ and $44.00 \%$. Both values are the number of hubs and bridges vs. the total infected population. Also, the number of bridge nodes was approximately equal to the number of hubs, so nearly every hub was interconnected. 


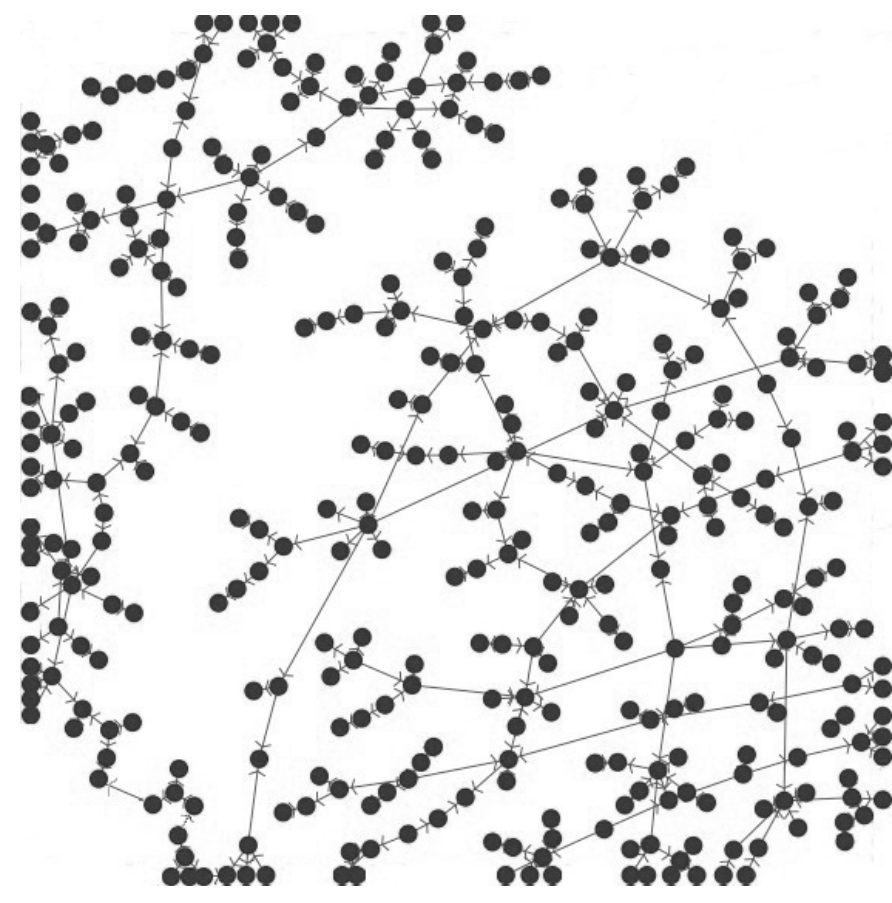

Fig. 3. The network map of the disease.

When the duration of the simulations are doubled, the number of people infected by the initial individual was similar, ranging from four to nine. The number of hubs and bridges versus the total infected population averaged $51.44 \%$ and $47.84 \%$ respectively. These numbers are very similar to the runs with shorter durations. When the simulation duration is doubled and the walking area size is increased to $2500 \times 2500$, the number of infected individuals that were in contact with the initial infected individual was also similar, ranging from four to nine compared to three to six in the shorter duration run. The number of hubs and bridges versus the total infected was most similar to the same population size in the smaller walk space, averaging $44.40 \%$ and $41.76 \%$.

Using real trace data, Yoneki et al. [10] showed that removing the top 100 hub nodes out of over 7500 nodes from consideration yields a significant reduction in the rate of epidemic spread. In the example network of Fig. 3, removal of seven highest degree hubs result in reduction over 30\% in the number of infected nodes. This is an important insight for the social network, which can be used to decide on the order of individuals to protect, to train or to separate from the network.

\section{CONCLUSIONS}

In this paper, we presented a series of systems which intend to lay the groundwork for modeling epidemic spreads using mobile devices. We developed a simulation model, using MATLAB, to evaluate the basic information needed to build an accurate mobile model and applied social networking metrics to assess the importance of each individual in the population. We also developed network maps to show an individuals position in a social network.
Further work in this area will include expanding the disease simulation to include real disease parameters and probabilities and porting the design to a mobile environment.

\section{REFERENCES}

[1] Severe Acute Respiratory Syndrome (SARS). [Online]. Available: http://www.cdc.gov/ncidod/sars/pdf/factsheet.pdf

[2] Updated CDC estimates of $2009 \mathrm{H} 1 \mathrm{~N} 1$ influenza cases, hospitalizations and deaths in the United States, April 2009 - April 10, 2010. [Online]. Available: http://www.cdc.gov/h1n1flu/estimates_2009_h1n1.htm

[3] New York Times health guide - Common Cold. [Online]. Available: http: //health.nytimes.com/health/guides/disease/common-cold/overview.html

[4] S. Wasserman and K. Faust, Social network analysis: methods and applications, ser. Structural analysis in the social sciences. Cambridge University Press, 1994. [Online]. Available: http://books.google.com/ books?id=CAm2DpIqRUIC

[5] Simulation environment for analysis and simulation. [Online]. Available: http://www.simulexinc.com/products/technology/

[6] EpiSimS: Epidemic simulation system. [Online]. Available: http: //www.lanl.gov/programs/nisac/episims.shtml/

[7] C. Barrett, R. Beckman, and K. Berkbigler, "TRANSIMS: Transportation analysis simulation," LAUR 00-1725, 2000.

[8] C. L. Barrett, K. R. Bisset, S. G. Eubank, X. Feng, and M. V. Marathe, "Episimdemics: an efficient algorithm for simulating the spread of infectious disease over large realistic social networks," in Proceedings of the ACM/IEEE conference on Supercomputing (SC), 2008, pp. 1-12.

[9] University of Cambridge FluPhone study. [Online]. Available: https: //www.fluphone.org/studyinfo.php

[10] E. Yoneki, P. Hui, and J. Crowcroft, "Wireless epidemic spread in dynamic human networks," vol. 5151, pp. 116-132, 2008.

[11] E. O’Neill, V. Kostakos, T. Kindberg, A. F. gen. Schieck, A. Penn, D. S. Fraser, and T. Jones, "Instrumenting the city: Developing methods for observing and understanding the digital cityscape," in International Conference on Ubiquitous Computing (UbiComp), ser. Lecture Notes in Computer Science, P. Dourish and A. Friday, Eds., vol. 4206. Springer, September 2006, pp. 315-332.

[12] T. Nicolai, E. Yoneki, N. Behrens, and H. Kenn, "Exploring social context with the wireless rope," in Proceedings of the Workshop MONET: LNCS 4277, 2006, pp. 874-883.

[13] R. Durrett, "Stochastic Spatial Models," SIAM Review, vol. 41, no. 4, pp. 677-718, 1999.

[14] D. T. Haydon, M. Chase-Topping, D. J. Shaw, L. Matthews, J. K. Friar, J. Wilesmith, and M. E. J. Woolhouse, "The Construction and Analysis of Epidemic Trees with Reference to the 2001 UK Foot-and-Mouth Outbreak," in Proceedings: Biological Sciences, vol. 270, no. 1511, 2003, pp. 121-127.

[15] S. Eubank, H. Guclu, V. S. A. Kumar, M. V. Marathe, A. Srinivasan, Z. Toroczkai, and N. Wang, "Modelling disease outbreaks in realistic urban social networks," Nature, vol. 429, no. 6988, pp. 180-184, 2004.

[16] V. Colizza, A. Barrat, M. Barthelemy, and A. Vespignani, "The role of the airline transportation network in the prediction and predictability of global epidemics," in Proceedings of the Natl. Acad. Sci. USA, vol. 7, no. 103, 2006, pp. 2015-2020.

[17] J. Broch, D. A. Maltz, D. B. Johnson, Y.-C. Hu, and J. Jetcheva, "A performance comparison of multi-hop wireless ad hoc network routing protocols," in Proceedings of the ACM/IEEE International Conference on Mobile Computing and Networking (MobiCom), October 1998, pp. 85-97.

[18] F. Bai and A. Helmy, "A survey of mobility models in wireless ad hoc networks," in Wireless Ad Hoc and Sensor Networks. Springer Verlag, October 2006, pp. 1-30.

[19] I. Rhee, M. Shin, S. Hong, K. Lee, and S. Chong, "On the Lévy-walk nature of human mobility," in Proc. of IEEE INFOCOM, April 2008, pp. 924-932.

[20] "MATLAB," The MathWorks Inc., Natick, MA, 2000.

[21] G. M. Viswanathan, S. V. Buldyrev, S. Havlin, M. da Luz, E. P. Raposo, and H. E. Stanley, "Optimizing the success of random searches," Nature, vol. 401, pp. 911-914, 1999.

[22] W. O. Kermack and A. G. McKendrick, "Contributions to the mathematical theory of epidemics, part i." vol. 115, no. 772. Proceedings of the Royal Society of London. Section A. Mathematics, Aug. 1927, pp. $700-721$. 